\title{
Microsurgical nerve root canal widening without fusion for lumbosacral intervertebral foraminal stenosis: technical notes and early results
}

\author{
Hisatoshi Baba, Kenzo Uchida, Yasuhisa Maezawa, Nobuaki Furusawa, Yasuhiro Okumura and Shinichi Imura \\ Department of Orthopaedic Surgery, Fukui Medical School, Shimoaizuki 23, Matsuoka, Fukui 910-11, Japan
}

\begin{abstract}
We describe a technique for microsurgical widening of the nerve root canal in the lumbosacral spine. We also report our early results in 31 patients (19 men and 12 women; average followup, 3.2 years) with such foraminal stenosis but without osseous defects in the pars interarticularis and/or spondylolisthesis. The affected nerve root was decompressed by interlaminar medial foraminotomy followed by lateral laminotomy and foraminotomy using a microsurgical technique. The procedure aimed at preserving bony continuity of the pars interarticularis and segmental motion. Neurological results showed a favourable.improvement of radicular symptoms and of intermittent claudication. The need for segmental fusion at the operated segment was excluded in 30 patients, but one patient subsequently required a transpedicular screw procedure with bone grafting. We recommend the present technique for effective decompression of the nerve root within the neural foramen, simultaneously maintaining lumbar spine mobile function.
\end{abstract}

Keywords: intervertebral foramen; stenosis; lumbosacral spine; surgical decompression; microsurgery; lumbar radiculopathy

\section{Introduction}

Osseous stenosis of the neural foramen is an uncommon cause of lumbar radiculopathy. ${ }^{1-3}$ This condition, in the so-called 'hidden zone' ${ }^{4}$ of the lumbosacral spine, in the absence of spondylolysis or spondylolisthesis showing bony abnormality of the pars interarticularis (isthmus), may cause impingement of the intraforaminal nerve root anteriorly, posteriorly, or circumferentially. The treatment of foraminal stenosis includes unilateral facetectomy or hemilaminectomy for nerve root release followed by fusion at the affected segment. However, if the nerve root canal is widened without such an extensive bony resection and bone grafting, it may be desirable for the lumbosacral spine so as to avoid a concomitant segmental fusion and to maintain mobility as close to the preoperative status as possible. We described a microsurgical nerve root canal widening technique for selected patients with radiologically confirmed lumbosacral intevertebral foraminal stenosis. This report describes our early results using this technique in 31 patients.
Correspondence: Hisatoshi Baba, MD, Associate Professor, Department of Orthopaedic Surgery, Fukui Medical School, Shimoaizuki 23, Matsuoka, Fukui 910-11, Japan

\section{Patients and methods}

Patient selection and clinical evaluation

Patients who had undergone microsurgical nerve root canal widening for lumbosacral foraminal stenosis with a minimum degree of 1 year follow-up were enrolled in the present study (average follow-up 3.2 years; range, 1 to 7.3 years). The cohort included 19 men and 12 women with an average age at operation of 49.1 years (range, 37 to 76 years). Patients presenting with symptomatic spondylolysis and isthmic or degenerative spondylolisthesis, showing osteocartilaginous nerve root compression with the neural foramen, were exlcluded from the study. These patients were treated with segmental fusion (posterolateral fusion and/or posterior lumbar intervertebral fusion) following extensive foraminal decompression. Prior to admission to our University Medical Centre, five patients had had one previous interlaminar operation, and one had undergone two interlaminar operations, without relief of leg pain and/or neurogenic claudication secondary to foraminal stenosis. It was clear that osseous foraminal stenosis was overlooked in the preoperative diagnosis in these six patients.

A modified assessment system based on that of An et $a l^{5}$ was used to evaluate the postoperative clinical status. This was scored as (1) excellent, complete recovery, with full return to previous activities; (2) good, neurologically improved but minimal leg 
discomfort continued without medication or rest; (3) fair, residual radicular symptoms necessitating the temporary use of medication and rest; and (4) poor, symptoms and signs unchanged or worsened.

\section{Radiological examination}

Diagnostic imaging often included multimodal techniques for assessing foraminal stenosis, determination of the level and site of nerve root compression, and the relationship between neural entrapment and osseous compression within the neural foramen. The diagnosis was established by a combination of radiological tests, including plain and three-dimensional (helical) computed tomography
(CT), selective nerve root infiltration test and radioculography, ${ }^{2}$ and magnetic resonance imaging (MRI). Based on radiological findings, we conveniently classified foraminal stenosis into three subtypes (Figure 1a, b). Type I lesion was associated with the presence of abnormal bone in the anterior border of the isthmus and the superior articular process impinging on the nerve root posteriorly. In type II lesion, bony bulge or osteophytes at the posterolateral vertebral corner compressed the nerve root primarily anteriorly, frequently associated with degenerated annulus fibrosus extrusion. Type III lesion represented a combination of types I and II lesions. All patients presented with varying degree of spondylosis, ie decrement in the intervertebral disc

a

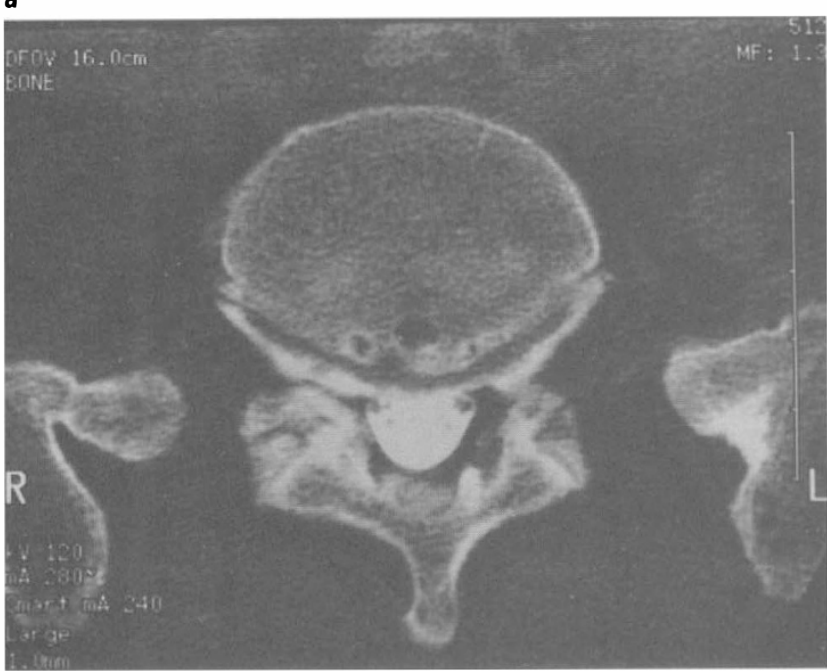

C
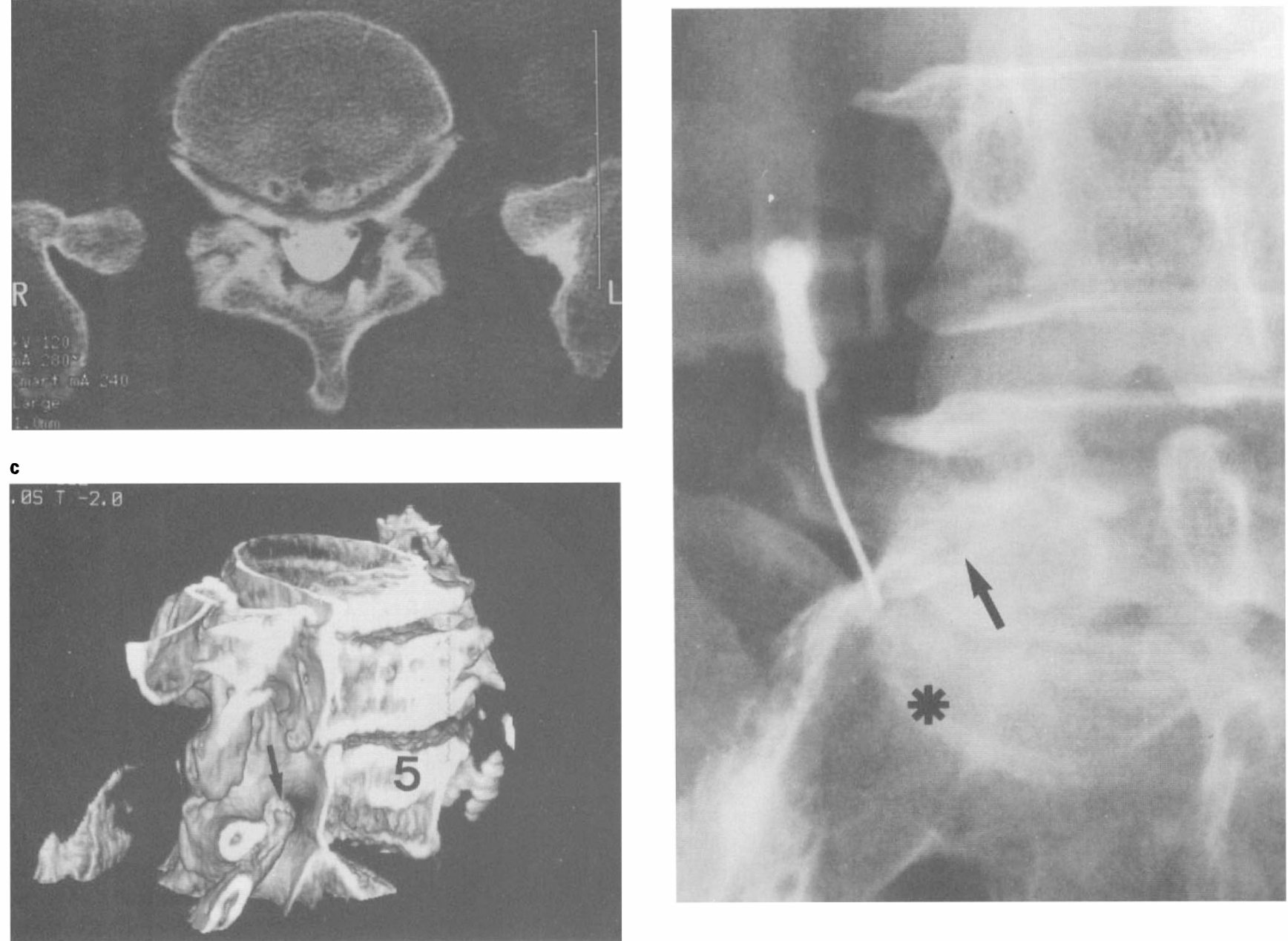

Figure 1 (a) Computed tomography of a patient with bilateral L5-S1 foraminal stenosis (type III). The nerve root canal is encroached on anteroposteriorly by the hypertrophied L5-S1 facet (superior articular process of S1) and sclerotic osseous bulge at the vertebral corner of L5. (b) Radiculography of a patient with type III foraminal stenosis at L5-S1 on the left, showing nerve root compression by the facet (arrow) and vertebral osteophytes (asterisk). (c) Split-sagittal display of three-dimensional computed tomography of a patient with type I foraminal stenosis at L5-S1 (5, L5 vertebra; arrow, superior articular process of S1 projecting within the neural foramen) 


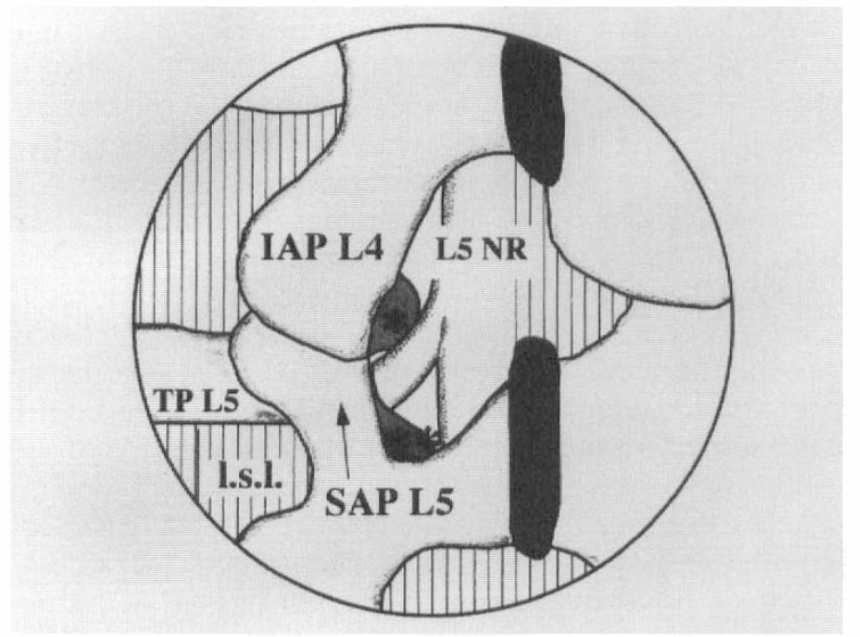

Figure 2 Schematic illustration showing the microscopic view of interlaminar medial fenestration and medial foraminotomy at L4-5 level on the left. The affected nerve root is decompressed through partial medial pediculotomy (asterisk) and relief from 'subarticular impingement' (double asterisk). L5 NR, the nerve root of L5; IAP and SAP, inferior and superior articular process; TP L5, transverse process of L5; and 1.s.1, lumbosacral ligament

height, vertebral osteophytes projecting into the spinal canal posteriorly, degenerative scoliosis, lordosis or kyphosis, hypertrophy and osteoarthritis of the facet joint adjacent to the affected nerve root. No clear anatomical correlation between the nerve root entrapment and these spondylotic changes were yet available in our patients, and, thus, consideration of this issue was excluded in this report.

The surgical indication included (1) prolonged symptoms and signs of lumbar radiculopathy, (2) positive selective nerve root infiltrating test and distinct evidence of nerve root compression on images of radiculography, (3) evidence of foraminal stenosis by plain and helical CT (Figure 1c), and (4) absence of apparent clinical instability. ${ }^{6}$ In the follow-up examination, the possible development of abnormal radiological findings suggestive of segmental instability at the operated segment was carefully evaluated. The operated segment was clinically unstable when the sagittal plane translation of the vertebra exceeded $3.5 \mathrm{~mm}$ (anterior slipping) and/or the anterior sagittal plane rotation increased by $>15^{\circ}$ (hypermobility) on lateral radiographs. An increase of scoliotic curvature (Cobb's angle $\geqslant 15^{\circ}$ ) was also considered as another sign of instability.

Two independent observers, other than the principal surgeon (HB), were involved in the clinical and radiological follow-up.

\section{Surgical technique}

In general, the nerve root canal widening technique consists of two parts; (1) interlaminar medial fenestra- a

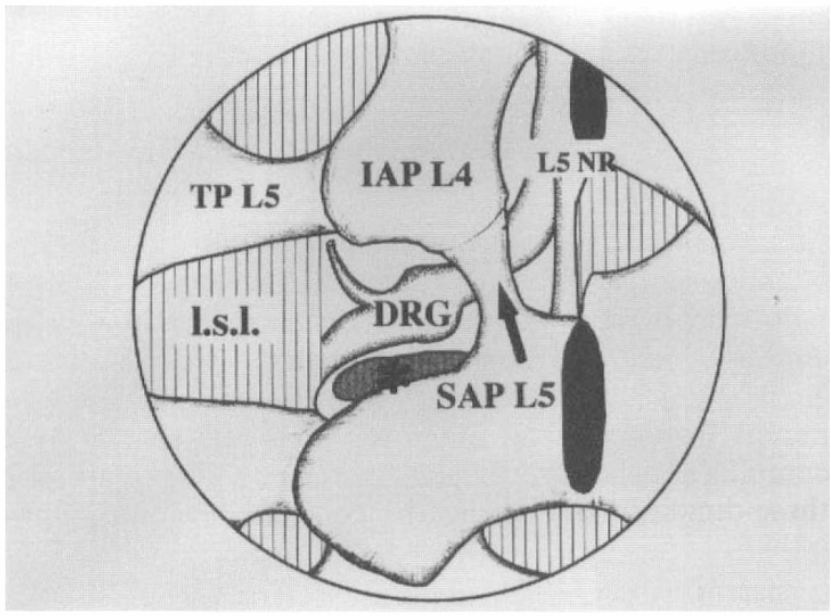

b

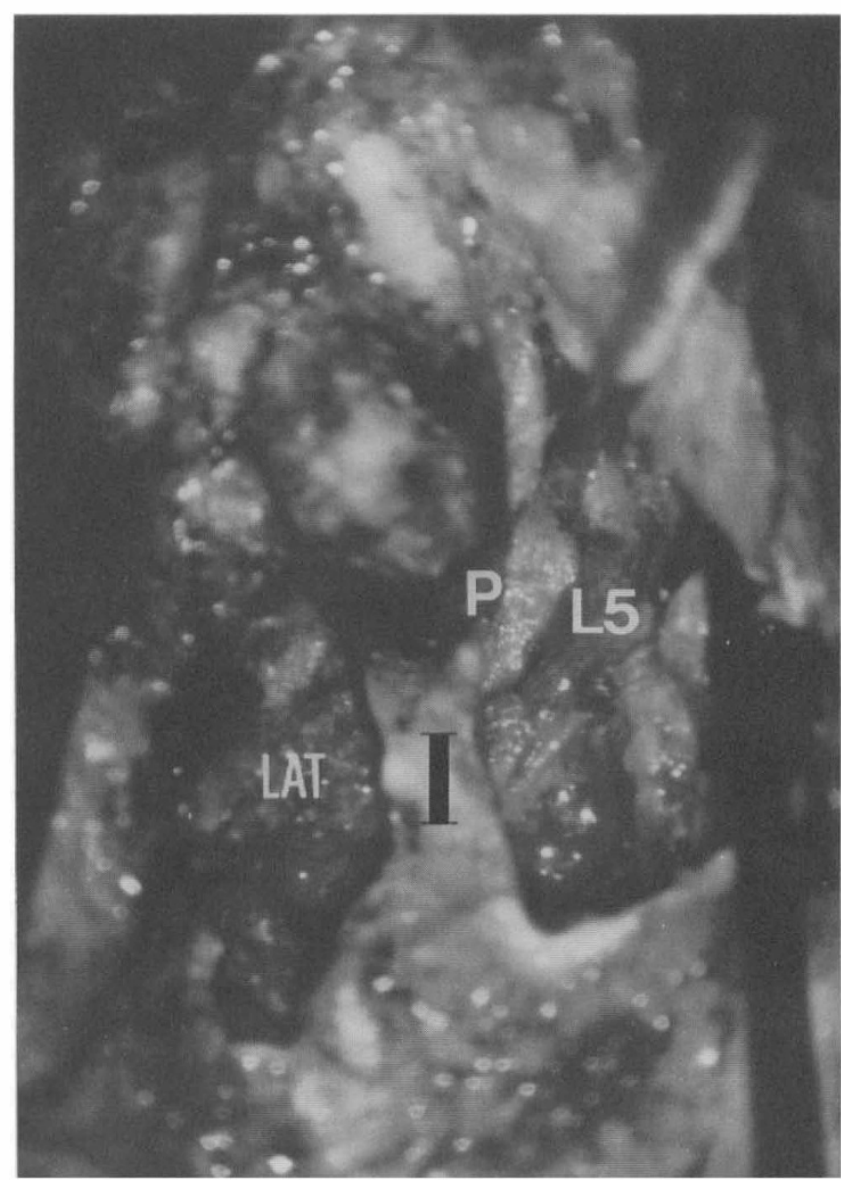

Figure 3 Schematic illustration and intraoperative microscopic photograph of the lateral fenestration and foraminotomy. (a) The dorsal root ganglion (DRG) and the exiting spinal nerve are decompressed following resection of vertebral osteophytes (asterisk) and lateral foraminotomy anterior to the superior articular process (SAP) of L5. Other abbreviations appear in Figure 2. (b) intraoperative microscopic photograph showing complete decompression of the L5 nerve root. P, Pedicle of L5; L5, the nerve root of L5; I, isthmus of the L5 lamina; LAT, lateral 


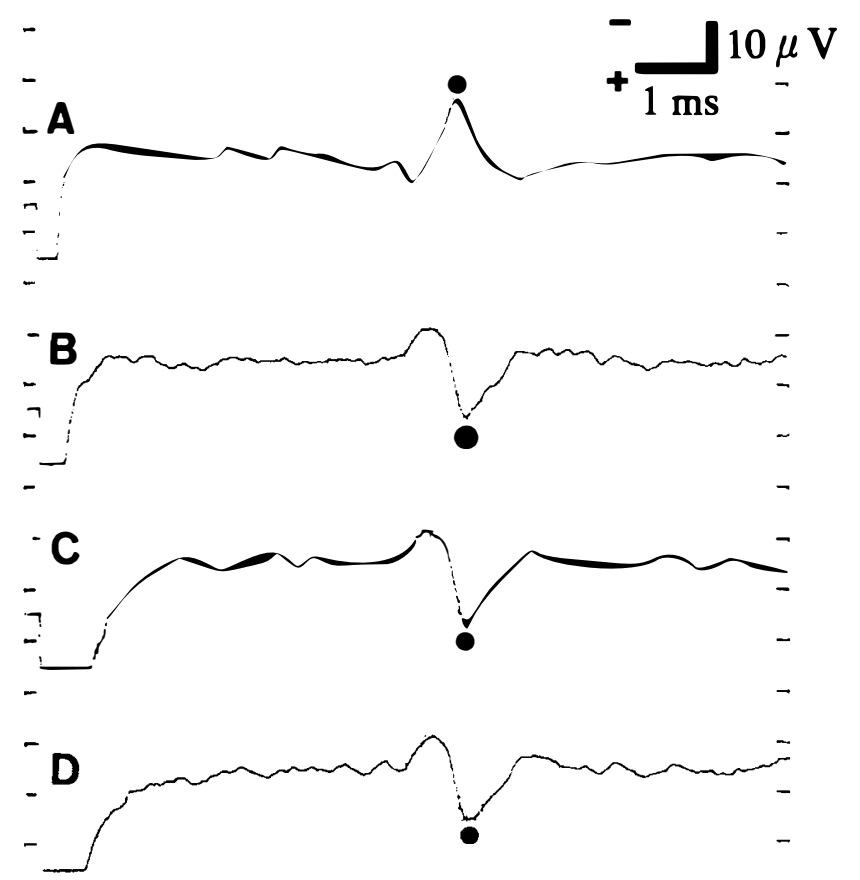

Figure 4 Nerve root action potentials recorded in a patient with type III foraminal stenosis at L4-5 level. Site A, Compression at a level just proximal to entrance to the neural foramen; site $\mathrm{B}$, site of compression caused by vertebral osteophytes and L5 superior articular facet within the neural foramen; site $\mathrm{C}$, at a level $5 \mathrm{~mm}$ distal to site $\mathrm{B}$; and site $\mathrm{D}$, at a level $10 \mathrm{~mm}$ distal to site $\mathrm{B}$. Positivedeflection evoked injury responses are present at sites B, C and D. Stimulation, at the epidural space of L1-2; modality of recording, orthograde and monopolar pick-up; stimulation, at 40 volts, rectangular pulse with $0.2 \mathrm{~ms}$ duration

tion and foraminotomy, and (2) microsurgical lateral laminotomy followed by circumferential neural decompression. This approach is essentially modified from that previously reported by Autrique et al, ${ }^{7} \mathrm{Jane}$ et $a l^{8}{ }^{8}$ and Fankhauser and de Tribolet, ${ }^{9}$ for the removal of intraforaminally herniated discs.

A midline skin incision is used and a Taylor retractor is placed just lateral to the facet joints above and below the affected neural foramen. Interlaminar fenestration is performed in a standard fashion but care is needed to identify 'pedicular kinking' and 'subarticular impingment', 2,4 at the entrance of the affected neural foramen. ${ }^{1}$ In patients with type I or III foraminal stenosis with 'subarticular entrapment', a small amount of bone is resected from the anteromedial border of the superior articular process to decompress the affected nerve root at the entrance of the neural foramen (Figure 2). As Beatty ${ }^{10}$ recommended, a fine-tipped 'foraminotomy rongeur' appears convenient to perform medial foraminotomy, but we prefer a high-speed diamond burr and a custom-made micro-Kerrison rongeur (with $2 \mathrm{~mm}$ blade and $1 \mathrm{~mm}$ thickness), because the anteromedial portion of the superior articular process is usually too hyperostotic and extremely hard to be resected by such a curette. In the presence of 'pedicular kinking', particularly on the concave side of degenerative scoliosis, a small amount of the inferomedial border of the pedicle can be resected (usually $1-2 \mathrm{~mm}$ width) with a high-speed burr to allow the nerve root to swing slackened. However, this maneuver is not essential for the medial foraminotomy. Approximately $25 \%$ of the isthmus width is resected medially to accomplish the medial foraminotomy, while a large amount of the posterior cortex of the isthmus should be preserved as much as possible to maintain its bony continuity.

The second step is decompression of the affected nerve root as well as the dorsal root ganglion following microsurgical lateral laminotomy and foraminotomy (Figure 3a). For this purpose, the lateral portion of the isthmus and then the inferior articular process are partially removed with a high-speed diamond burr. The extent of bony resection varies from one patient to another and is determined by the degree of degenerative hypertrophy of the facet joint located caudal to the affected nerve root, but in most cases we preserve more than one-half of the transverse diameter of the isthmus. The anterior cortex of the isthmus facing the affected nerve root is removed by a high-speed diamond burr tilted obliquely or by using a curved foraminotomy rongeur. This step is essential for type I and III lesions. The ligamentum flavum, already torn during medial foraminotomy, is then identified. From this level, the ligamentum flavum is resected laterally using a Kerrison rongeur followed by full exposure of the dorsal root ganglion within the adipose tissue. Care is needed to explore the nerve root and dorsal root ganglion. The latter is usually stuck within the neural foramen by the hypertrophied facet joints rostrally and caudally. Bluntly retracting the nerve root and dorsal root ganglion cranially, vertebral osteophytes seen in type II and III lesions at the exiting zone of the neural foramen can be removed using a high-speed diamond burr (Figure $3 b$ ) or a commerically available impactor. However, this procedure requires utmost attention so as to avoid application of a dangerous retraction force or afflicting mechanical injury to the nerve root.

During the nerve root and spinal nerve decompression, we often measure nerve root action potentials to confirm the actual level of neural compression (Figure 4 ), but this examination is not essential for the surgical technique itself. A free fat graft is placed around the nerve root and dorsal root ganglion within the neural foramen, and a soft suction drain (eg Jackson drain) is positioned before wound closure.

In the present series, the time of operation ranged from 1.8 to $3.3 \mathrm{~h}$, with a mean of $2.6 \mathrm{~h}$. The blood loss averaged $84 \pm 29 \mathrm{ml}$ (mean \pm standard deviation; range, 45 to $190 \mathrm{ml}$ ). The patient is allowed to sit and then ambulate 3 or 4 days after surgery with a lumbosacral spine brace and return to normal levels of activity 2 to 3 months later. 


\section{Summary of early results}

Spinal levels affected and types of foraminal stenosis The affected spinal level was L3-4 in four patients (13\%), L4-5 in 19 patients (61\%), and L5-S1 in eight patients $(26 \%)$. All patients had single nerve root involvement, except in one case with L5-S1 involvement bilaterally (Figure 1a). Ten patients were assessed to have type I lesion, six with type II lesion, and 15 patients had type III lesion. While the L4-5 level was the most frequently involved region, no remarkable correlation was found between the type of stenosis and the affected spinal level.

\section{Clinical presentation and radiological follow-up}

Demographic data of clinical presentation according to the type of foraminal stenosis are summarised in Table 1. Twenty-six $(84 \%)$ patients complained of lower back and gluteal spasm and/or pain preoperatively. Radicular pain was reported by 24 patients even at resting position. Mechanical signs were observed in 14 patients before surgery and a variable degree of neurogenic intermittent claudication was reported by $17(55 \%)$ patients. Neurological effects were more prominent in the sensory system than in motor function.

At follow-up, about $50 \%$ of patients still complained of low back discomfort, mostly with decreased magnitude, but the recovery from radicular pain and intermittent claudication was remarkable. A total of 11 patients (five patients with type I lesion, one with type II lesion, and five with type III lesion) were found to have decreased level of neurologic signs. Functional outcome was assessed as excellent or good in 25 patients, and fair or good in six patients.
No patient developed lumbar spine instability, as defined by our radiological criteria. In one of two patients with poor outcome (one with type II lesion and another with type III lesion), presenting with severe spondylosis at the L3-4 level postoperatively, required a salvaging transpedicular screw arrangement to eliminate possible 'facet-induced' low back pain, 2 years after the nerve root canal widening operation.

\section{Surgery-related complications}

Two patients reported a temporary increase in dysesthesias or parasthesias along the affected nerve roots (one in L4 and another in L5 root distribution) in the first postoperative week, but both recovered spontaneously by the end of two postoperative weeks. No other complications, such as wound infection or haematoma, cerebrospinal fluid leakage, or fracture of the isthmus, occurred in our patients.

\section{Discussion}

Bony compression of the nerve root in the lumbosacral neural foramen, in the absence of isthmic spondylolysis and/or spondylolisthesis, is possible, but appears to be less recognised in comparison with usual spinal canal stenosis. ${ }^{1,2,11}$ Although current-generation CT and MRI are helpful in identifying intraforaminal pathology, it is not always easy to confirm symptomatic foraminal neural entrapment requiring surgical decompression. When surgery is indicated, it may be a generally accepted idea that the affected nerve root should be decompressed through an extended bony resection, such as unilateral facetectomy or transpars foraminal decom-

Table 1 Types of foraminal stenosis and patient clinical data

\begin{tabular}{|c|c|c|c|c|c|c|}
\hline & \multicolumn{2}{|c|}{ Type $I(\mathrm{n}=10)$} & \multicolumn{2}{|c|}{ Tyре $I I(\mathrm{n}=6)$} & \multicolumn{2}{|c|}{ Type III $(\mathrm{n}=15)$} \\
\hline & before surgery & follow-up & before surgery & follow-up & before surgery & follow-up \\
\hline \multicolumn{7}{|l|}{ Symptoms } \\
\hline stiffness and low back spasm & 10 & 5 & 4 & 4 & 12 & 4 \\
\hline numbness & 8 & 3 & 4 & 2 & 8 & 2 \\
\hline weakness & 4 & 1 & 3 & 1 & 9 & 2 \\
\hline radicular pain during rest & 7 & 1 & 6 & 1 & 7 & 1 \\
\hline \multicolumn{7}{|l|}{ Claudication } \\
\hline$<100$ metres & 2 & 0 & 1 & 0 & 3 & 0 \\
\hline $100-200$ metres & 4 & 1 & 2 & 0 & 3 & 1 \\
\hline $200-500$ metres & 1 & 0 & 0 & 0 & 1 & 0 \\
\hline \multicolumn{7}{|l|}{ Neurologic Signs } \\
\hline mechanical signs* & 5 & 1 & 3 & 0 & 9 & 2 \\
\hline motor deficit (L3, L4, L5) & 1 & 1 & 1 & 0 & 2 & 1 \\
\hline sensory deficit (L3, L4, L5) & 6 & 2 & 4 & 1 & 7 & 1 \\
\hline deep tendon reflex changes $\ddagger$ & 1 & 1 & 1 & 0 & 2 & 1 \\
\hline \multicolumn{7}{|l|}{ Outcome Assessment } \\
\hline excellent + good & & 7 & & 5 & & 13 \\
\hline fair + poor & & 3 & & 1 & & 2 \\
\hline
\end{tabular}

*mechanical signs: straight-leg raising test $<50^{\circ}$ and positive femoral nerve stretch test; $\ddagger$ deep tendon reflex changes, abnormal patellar and Achilles responses 
pression. ${ }^{12}$ After such an extensive decompression, it is in fact easy to mechanically stabilise and fuse the affected segment with hardware use. However, one wonders if complete decompression of the nerve root is possible without concomitant segmental fusion/ stabilisation, in order to preserve functional mobility at the affected segment. It is important to be concerned with the long-term adverse effect of segmental fusion on the adjacent spinal levels, so that the present 'non-fusive' and 'mobility-maintaining' techniques for foraminal decompression may be expected to be one recommended surgical option.

The presence of foraminal stenosis is confirmed only when compression of the nerve root by the osseous elements within the neural foramen is demonstrated radiologically. Radiological diagnosis of foraminal stenosis is infrequently difficult even by currentgeneration CT and MRI, however. Recently, Smith et $a l^{13}$ and Zinreich ${ }^{14}$ have recommended the use of helical CT scan to assess intraforaminal bony lesions and stenosis. Foraminal space narrowing can be visualised with helical CT images, using bone windows, soft tissue windows, and sagittal reformations. As Smith et al ${ }^{13}$ have advocated, such threedimensional display is also helpful in planning the surgical technique. We were not able to perform such stereotaxic assessment routinely, but in selected cases, multiplanar sagittal and coronal images as well as three-dimensional CT reconstructions were of use for determining osseous lesions within the neuronal foramen. However, possibly due to a variable degree of spondylosis at the affected level, we are not yet able to quantify differences in the canal size between healthy people and patients. On the other hand, transaxial and parasagittal MRI may also be helpful in evaluating the degree and site of nerve root compression within the neural foramen. However, as Zinreich ${ }^{14}$ has pointed out, severe neural compression is frequently present on MRI despite benign or sometimes no clinical features. Furthermore, each plane of MRI demonstrates cross-sectional image of the nerve root subjected to complicated osseous as well as ligamentous compression within the neural foramen. As Epstein et $a l^{15}$ have also pointed out, it should be remembered that a high variability of associated structural (bony) abnormalities in the foraminal and far lateral area of the lumbar spine is observed in elderly patients. Therefore, at present time, we believe that radiological diagnosis of foraminal stenosis appears to be accomplished on individual basis by CTs and MRIs. Selective nerve root infiltration technique with radiculography is one method that offers a good visualisation of the affected nerve root and the exiting lumbar spinal nerve. It often shows the anatomical deviation of the nerve root within the neural foramen. ${ }^{2}$ We prefer this technique for anteroposterior assessment of the affected nerve root, but the findings of radiculography must be carefully compared with those of transaxial as well as helical CT images during surgical planning. This method, however, is rather technically demanding so that its indication and performance should be carefully considered.

The common surgical approach for foraminal stenosis may be unilateral facetectomy ${ }^{16}$ or transpars address $^{12}$ followed by fusion/stabilisation at the operated segment. The use of transpedicular screws after such extensive decompression certainly ensures an immediate postoperative spine stability and facilitates early ambulation. While we think the use of this technique is one option in the surgical management of foraminal stenosis, we believe that a segmental fusion may be avoided if the operated segment can be maintained mechanically stable and functional. ${ }^{17}$ For preservation of mechanical stability, it is essential to widen the neural foramen without unnecessary resection of a large section of the bone surrounding the nerve root within the neural foramen. The current technique is basically a refined method of Autrique et $a l^{7}$ and by Jane et $a l^{8}$ for removing foraminal herniated intervertebral discs, without a concomitant lumbar spine fusion. As these workers suggested, when the compressive lesion is removed from the medial and lateral approaches, it may be a good surgical option, saving a fusion technique.

For further enhancing the safety of our 'fusionsaving' surgical modification, the use of microsurgical technique is recommended, as is often employed in the excision of far-lateral herniated intervertebral disc. $^{18-20}$ In the current series of cases, the dorsal root ganglion and the exiting spinal nerve were frequently found to be confined within the neural foramen by two pedicles, vertebral osteophytes, with a bulged annulus fibrosus, and a hypertrophied facet joint (superior articular process) caudally. Furthermore, in the far-lateral area, the hypertrophied synovial tissue in the degenerated facet joint may hinder a safe exposure and decompression of the affected nerve. The microsurgical approach would assist in safe decompression by minimising the risks of nerve injury during lateral foraminotomy. Darden et $a l^{20}$ however, have recently reported a transient aggravation of dysesthesias following microsurgical resection of far-lateral herniated discs despite a very gentle manipulation of the nerve root ganglion and the exiting spinal nerve. In the present series, two $(6 \%)$ patients complained of temporary increase of paraesthesia in areas innervated by the affected nerve roots, possibly due to unintended manipulation of the ganglion. We suggest exercising utmost care during resection of vertebral osteophytes in type II or III lesions, the nerve root ganglion should be retracted caphalad during the decompression.

Although our preliminary results appeared neurologicaly favourable, we are still uncertain how much isthmus must remain to assure biomechanical stability between the two facet joints. To our knowledge, there are no reports that have discussed this issue, however. It is generally believed that at least one-half of the facet joint is necessary for maintaining its biomecha- 
nical function after partial medial facetectomy for ordinary lumbar spinal canal stenosis. In most cases, we have thus attempted to preserve at least the middlehalf of the isthmus and the medial portion of the inferior articular process. At follow-up, there was no fracture at the partially resected isthmus and, thus, our technique may not be associated with future biomechanical problems, even though the suggestion is empirical. In this regard, Chen et $a l^{21}$ recently described a small degree of bone re-growth at the laminotomy site in the lumbar spine. This biological reaction is favourable for the isthmus to be mechanically strengthened. However, this suggestion warrants further evaluation based on long-term radiological follow-up.

We conclude that microsurgical nerve root canal widening via interlaminar medial foraminotomy and lateral laminotomy, without concomitant segmental fusion/stabilisation, is a useful surgical option for patients with symptomatic lumbosacral foraminal stenosis.

\section{References}

1 Lee CK, Wolfgang R, William G. Lateral lumbar spinal canal stenosis: classification, pathologic anatomy and surgical decompression. Spine 1988; 13: 313-320.

2 Kunogi J, Hasue M. Diagnosis and operative treatment of intraforaminal and extraforaminal nerve root compression. Spine 1991; 16: $1312-1320$.

3 Stephens MM, Evans JH, O'Brien JP. Lumbar intervertebral foramens: an in vitro study of their shape in relation to intervertebral disc pathology. Spine 1991; 16: 525 - 529.

4 Macnab I. Negative disc exploration: an analysis of the causes of nerve root involvement in sixty-eight patients. J Bone Joint Surg Am 1971; 53: 891 - 903 .

5 An HS, Vacaro A, Simeone FA, Balerston RA, O'Neil D. Herniated lumbar disc in patients over the age of fifty. $J$ Spinal Disord 1990; 3: 143 - 146.

$6 \mathrm{Baba} \mathrm{H}$ et al. Posterior limbus vertebral lesions causing lumbosacral radiculopathy and the cauda equina syndrome. Spinal Cord 1996; 34: $427-432$.
7 Autrique A et al. Le traitement chirurgical des hernies discales foraminales lombaires. Intérêsts et indications de la vois combinée interlaminaire at extraarticulaire. J Chir (Paris, 1989; 126: $338-343$.

8 Jane JA et al. A neurosurgical approach to far-lateral disc herniation: technical note. J Neurosurg 1990; 72: 143-144.

9 Fankhauser H, de Tribolet N. Extraforaminal Approach fol Extreme Lateral Lumbar Disc Herniation. In: Torrens MJ Dickson RA (ed). Operative Spinal Surgery. Churchill Living stone: Edinburgh, 1991, pp 145-160.

10 Beatty R. Foraminotomy rongeur. Spine 1991; 16: S295-S297.

11 Major HM, Helms CA. Central and foraminal stenosis of thi lumbar spine. Neuroimaging Clin North Am 1993; 3: 557-566.

12 Epstein NE. Different surgical approaches to far lateral lumba disc herniations. J Spinal Disord 1995; 8: 383-394.

13 Smith GA, Aspden RM, Porter RW. Measurement of vertebra foraminal dimensions using three-dimensional computerizer tomography. Spine 1993; 18: 629-636.

14 Zinreich SJ. Three-dimensional Computed Tomography of th Spine. In: Roland LR (ed). Spine: State of the Art Review; Spina Imaging. Hanley \& Belfus: Philadelphia, 1995, pp 287-297.

15 Epstein NE, Epstein JA, Carras R, Hyman RA. Far latera lumbar disc herniations and associated structural abnormalities an evaluation in 60 patients of the comparative value of $\mathrm{CT}$ MRI, and myelo-CT in diagnosis and management. Spine 1990 15: $534-539$.

16 Garrido E, Connaughton PN. Unilateral facetectomy approacl for lateral lumbar disc herniation. J Neurosurg 1991; 74: 754 756.

17 Baba H, Imura S, Tomita K. Surgical strategy and results o lumbar spinal canal stenosis. Abstract read at the Sixtl International Conference of Lumbar Fusion and Stabilizatior (ICLFS). Canberra, Australia, November 2-5, 1993.

18 Reulen HJ, Pfaundler S, Ebelling U. The lateral microsurgica approach to the 'extracanalicular' lumbar disc herniation. Act, Neurochir (Wien) 1987; 84: 64-67.

19 Maroon JC et al. Diagnosis and microsurgical approach to farlateral disc herniation in the lumbar spine. J Neurosurg 1990; 72: $378-382$.

20 Darden BV II et al. Far lateral disc herniations treated by microscopic fragment excision: techniques and results. Spint 1995; 20: $1500-1505$

21 Chen $\mathrm{Q}$ et al. Postoperative bone regrowth in lumbar spinal stenosis: a multivariate analysis of 48 patients. Spine 1994; 19: $2144-2149$ 